\title{
Eine Methode zur Gewinnung abnorm großer Protoplasten.
}

\author{
Von \\ Erust Küster \\ in Kiel. \\ Eingegangen am 31. Januar 1910.
}

Bei den Versuchen, das Zellenleben der Pflanzen und der in den Zellen schlummernden Fähigkeiten zu Wachstum, Gestaltung usw. experimentell zu erforschen, bestand die Aufgabe in der Mehrzahl der Fälle darin, durch planmäßige Änderungen in den äußeren Lebensbedingungen der Zelle und durch Studium der Reaktionen, mit welchen die Zelle etwa auf den Wechsel der sie beeinflussenden äußeren Faktoren zu antworten vermag, zur Lösung des Problems beizutragen. Viel seltener gelang es, durch bestimmte experimentelle Eingriffe die Konstitution der Zelle und damit die wirksamen sinneren " Bedingungen in kontrollierbarer Weise zu verändern.

Die Zahl der Methoden, die für diese Arbeitsrichtung in Betracht kommen, ist leider eine sehr beschränkte; die beiden wichtigsten sind wohl folgende:

Durch Plasmolyse gelingt es, an Pflanzenzellen sehr verschiedener Art den Plasmaleib in zwei oder mehr Teilstücke zu zerlegen; liegen Versuchspflanzen mit einkernigen Zellen vor, so kann natürlich nur in einem der durch Plasmolyse gewonnenen Plasmasticke der Kern verblieben sein, und wir haben Gelegenheit, das Verhalten des kernhaltigen Plasmaballens mit dem des kernlosen zu vergleichen $\left.{ }^{1}\right)$.

Methoden, um Zellen mit abnorm reichlicher Kernmasse zu gewinnen, hat GerassimofF beschrieben: wenn man Spirogyra-Zellen,

1) Vgl. besonders KLeBs, G., Beiträge zur Physiologie der Pflanzenzelle. Untersuch. Bot. Inst. Tübingen. Bd. II. 1888. S. 489. Literatur bei Küster, E., Aufgaben und Ergebnisse der entwicklungsmechanischen Pfanzenanatomie. Progressus rei botan. Bd. II. 1908. S. $502 \mathrm{ff}$. 
die gerade im Begriff sind sich zu teilen, und deren Kern bereits in zwei Tochterkerne sich zerlegt hat, durch Abkühlung oder durch Behandlung mit anästhetischen Mitteln im normalen Fortgang des Zellteilungsprozesses stört, so resultieren schließlich oft Zellen, in welchen sich beide Tochterkerne vereinigen, während die andre Tochterzelle kernlos ausgeht. Die Zellen der ersten Art sind somit zu einem Überschuß an Kernsubstanz gekommen; die Nuclearmasse steht zu der des Cytoplasmas in einem andern Verhältnis als in snormalen " Zellen. Gerassmofy hat das Schicksal dieser kernsubstanzreichen Zellen verfolgt und hat gezeigt, daß aus ihnen besonders großzellige Fäden erwachsen ${ }^{1}$ ).

Ich nannte diese Methoden die beiden wichtigsten, weil bisher ihre Anwendung die meisten Resultate gezeitigt hat. Ihre Anführung mag genügen, um an zwei, Beispielen zu zeigen, in welchem Sinne oben von einer Veränderung der Konstitution der Zelle und einem experimentell herbeigeführten Wechsel der »inneren * Lebensbedingungen die Rede war.

Die Plasmolyse, durch welche der Protoplast einer Zelle von der ihn sonst umschließenden Cellulosehaut frei und für den experimentierenden Mikroskopiker zugänglich und handlich gemacht wird, wird uns - abgesehen von der oben erörterten Methode - gewiß noch zu mancherlei Möglichkeiten des experimentellen Eingriffs ins Zellenleben verhelfen. Von einer neuen Art derartiger Experimente soll im folgenden die Rede sein.

Wenn man Schnitte durch geeignete Pflanzenorgane in starken Salzlösungen plasmolysiert und dann durchschneidet, so trifft der Schnitt hier und da auf die Zellen derart, daß das vom Plasma nicht mehr ausgekleidete Cellulosegehäuse angeschnitten wird, der Protoplast selbst aber intakt bleibt. Die Aussichten, derartig geöffnete Zellen mit unverletztem Plasmaleibe zu erhalten, werden um so ginstiger sein, je stärker sich die Protoplasten kontrahiert haben und je größer daher der Raum zwischen ihnen ist, den das trennende Messer treffen darf, ohne das Plasma zu zerstören. Besonders günstig liegen die Verbältnisse offenbar dann, wenn anisodiametrische, sehr langgestreckte Zellen vorliegen, in welchen sich die Protoplasten vorwiegend oder ausschließlich in einer Richtung kontrabieren, -

1) Vgl. Gerassimofr, J. J., Über den Einfluß des Kerns anf das Wachstum der Zelle. Bull. Soc. imp. Natur. de Moscou. 1901. No. 1 u. 2. Die Abhängigkeit der Größe der Zellen von der Menge ihrer Kernmasse. Zeitschr. f. allgem. Physiol. Bd. I. 1902. S. 220. 
eben in der Längsrichtung der Zelle; Präparate mit intakten Protoplasten werden sich dann erzielen lassen, wenn man senkrecht zu dieser Richtung den plasmolysierten Gewebefetzen durchtrennt.

Aus diesen und andern Grüden arbeitete ich vorzugsweise mit den Epidermen verschiedener Monokotyledonen.

Läßt man zu Präparaten, in welchen angeschnittene Zellen mit intakten Protoplasten vorliegen, langsam Wasser zutreten, so dehnen sich die Protoplasten in bekannter. Weise aus und strecken sich zu dem angeschnittenen Zellwandgehäuse hervor. Bei verschiedenen Pflanzen verhält sich der Inhalt der Zellen verschieden: bei manchen schlüpfen die Plasmaleiber leicht und ohne Unterbrechnng ihrer Vorwärtsbewegung aus, etwa wie große Schwärmsporen aus dem geöffneten Zoosporangium (z. B. die Zellen der Epidermis von Tulpenzwiebelschalen, morphologische Unterseite!), - bei andern werden sie durch mehr oder minder zahlreiche dünne Plasmafäden an der Zellenhaut und im Innern der angeschnittenen Zellen zurückgehalten (z. B. bei entsprechenden Präparaten von Allium Cepa): man lernt es aber leicht, das .Ansschlüpfen der Protoplasten durch leichten Druck mit der Präpariernadel auf das Deckglas oder durch sanftes Klopfen zu beschleunigen.

Eine Methode zur Gewinnung abnorm großer Protoplasten wird dadurch ermöglicht, $d a \beta$ die aus verschiedenen Epidermiszellen - ich schildere hier das Verhalten der von Allium Cepa gewonnenen Präparate - ausgetretenen Protoplasten sich miteinander zur Vereinigung bringen lassen. Allerdings bedarf es einiger einfacher Handgriffe, um auch ohne besonderen Zeitaufwand eine derartige Fusion zu erhalten. Empfehlenswert ist es, die durch einen scharfen Rasiermesserschnitt gewonnenen Präparathälften dicht aneinander zu legen: der zwischen den beiden Hälften klaffende Spalt hält die ausgetretenen Plasmakugeln gefangen und erleichtert es, sie zur Fusion zueinander za führen; ferner empfiehlt es sich, die beiden Schnitthälften etwas gegeneinander zu verschieben, so daß nirgends die zusammengehörigen Teilstïcke der einzelnen Epidermiszellen einander gegenuiberliegen: man hat dann begrindete Aussicht, daß hier und da unmittelbar einander gegenüber je zwei Protoplasten aus angeschnittenen Zellen hervortreten; man gewinnt anf diese Weise am sichersten Fusionen, noch bevor die Protoplasten völlig aus dem Zellenlumen hervorgeschlüpft sind.

Ist das Ergebnis, daß beliebige vegetative Zellen aus einem Präparat wie der hier geschilderten $Z_{w}$ wiebelepidermis im Experiment 
zur Fusion gebracht werden können, an sich schon von zellenphysiologischem Interesse, so läßt sich ferner hoffen, daß die weitere Beobachtung der künstlich erzielten, abnorm großen, mehrkernigen Protoplasten die Behandlung protoplasmamechanischer Fragen gestatten wird.

Wir können mit unsrer Versuchstechnik noch weiter gehen und die Beschaffenheit der erzielten Riesenprotoplasten insofern variieren, als es bei einiger Übung leicht gelingt, die austretenden Protoplasten willkürlich zu zerstückeln und zu zerkleinern: wenn man Protoplasten, welche eben im Begriff sind, aus der Öffnung der abgeschnittenen Zellen vorzutreten, durch Druck mit der Präpariernadel auf das Deckglas aus dem Zellenlumen weiter herauspreßt und dann den Druck plötzlich abnehmen läßt, so zerreißt der Plasmaleib oft, und die austretenden Teilstuicke verhelfen uns bei Fusion mit einem ungeteilten Plasmaleibe zu Protoplasten von abnormer Größe, bei welchen aber das Massenverhältnis zwischen Cytoplasma und Zellenkern nicht mehr das normale ist, sondern von der Norm in der einen oder andern Richtung abweicht. Einiger Geduld bedarf es allerdings, um Fusionen dieser Art zu erhalten.

Die Schattenseite dieser und aller ähnlicher zellenphysiologischer Arbeitsmethoden liegt darin, dạß sie immer nur bei Objekten bestimmter Art anwendbar sind. Das gilt auch für die hier beschriebene. Sehr viele Pflanzenzellen werden nämlich durch die für uns unentbehrliche Prozedur der Plasmolyse in der Weise beeinfluBt, daß isolierte Plasmastücke ein und derselben Zelle sich auch bei gegenseitiger Berührung und selbst dann, wenn sie sich gegenseitig. kräftig drücken und deformieren, nur schwer oder gar nicht mebr miteinander vereinigen. Offenbar handelt es sich um physikalische und chemisch-physikalische Veränderungen in der Oberflächenbeschaffenheit der Plasmakugeln ${ }^{1}$. Namentlich ferner ist daran zu denken, daß diese der Fusion ungünstigen Veränderungen bei Anwendung verschiedener plasmolysierende Mittel sich in verschieden hohem Grade bemerkbar machen. Als ein für unsrè Zwecke geeignetes Plasmolyticum kann ich Calciumnitratlösungen nennen; ich operierte vorzugsweise mit Normallösungen. Epidermisprotoplasten von Allium Cepa fusionieren nach Plasmolyse mit solchen sebr leicht: schon bei flüchtiger Berührung, die nur Bruchteile einer Sekunde

1) Vgl. Küster, E., Über die Verschmelzung nackter Protoplasten. Ber. (l. D. Bot. Ges. Bd. XXVII. H. 10. 1909. S. 589 . 
währt, verkleben die sich berührenden Plasmakugeln derart, daß sie sich dann, wenn man sie wieder voneinander entfernt, za feinen Spitzchen ausziehen, die bald wieder von der Hauptmasse der Plasmakugel aufgenommen werden. Bei länger währender Berührung vereinigen sich die beiden Protoplasten meist sehr bald za einem einzigen.

Meine Untersuchungen über das physiologische Verhalten der durch Fusion künstlich gewonnenen Protoplasten abnormer Größe sind noch nicht abgeschlossen und sollen später an andrer Stelle ausführlichere Besprechung finden. Auf dieselbe Gelegenheit mag ein Vergleich der an Pflanzenzellen beobachteten Erscheinungen mit den von tierischen Eiern her bekannten ${ }^{1)}$ verspart bleiben.

Kiel, Botanisches Institut der Universität, Januar 1910.

1: Vgi. Driesch, H., Studien über das Regenerationsvermögen der Organismen. IV. (Arch. f. Entw.-Mech. 1900. Bd. 10. S. 411). Drei Aphorismen zur Entwicklungsphysiologie jüngster Stadien (Arch. f. Entw.-Mech. 1903. Bd. 17. S. 41). LiLriE, F. R., Differentations without cleavage in the egg of the annelid Chactopterus pergammitacus (Arch. f. Entw.-Mech. Bd. 14. 1902. S. 477, 